hep-th/9907036

\title{
Some comments about Schwarzschild black holes in Matrix theory
}

\author{
J. Klusoň F $^{-2}$ \\ Department of Theoretical Physics and Astrophysics \\ Faculty of Science, Masaryk University \\ Kotlárská 2, 611 37, Brno \\ Czech Republic
}

\begin{abstract}
In the present paper we calculate the statistical partition function for any number of extended objects in Matrix theory in the one loop approximation. As an to application, we calculate the statistical properties of $\mathrm{K}$ clusters of Do branes and then the statistical properties of $\mathrm{K}$ membranes which are wound on a torus .
\end{abstract}

${ }^{*}$ E-mail:klu@physics.muni.cz 


\section{INTRODUCTION}

Matrix theory [1] is one of the most promising ideas for a nonperturbative definition of $\mathrm{M}$ theory. There are many hints that confirms the validity of this conjecture, for example, this theory can reproduce the leading order scattering amplitude for gravitons in eleven dimensional low energy supergravity without momentum transfer in the longitudinal direction (for example 2 (4). We also have a good knowledge of what happens when one compactifies this theory on a $p$ dimensional torus for $p<6$. [5 9] This theory also reproduces all five string theories 10 14]

On the other hand, there are many unresolved problems. Eleven dimensional Lorenz invariance is still mysterious, the theory is defined in a fixed background. Processes with longitudinal momentum transfer between gravitons has not been calculated and this process would be rather useful for proving Lorenz invariance. The other serious problem is that matrix theory is not well understood for more complicated backgrounds (for example the $p$-torus with $p>6$ ).

One of the most interesting problems where matrix theory can be tested is the quantum mechanical description of black holes. This is because matrix theory, as a candidate for a nonperturbative definition of M theory, must be able to describe these black holes and indeed this have been done in many papers (for example [15], 24]). In these approaches, a remarkably simple model for Schwarzschild black holes was presented in that the Schwarzschild black hole is described as a collection of $N$ D0 branes, and the basic properties of these black holes comes from a statistical study of this system. In this approach it is important to have these branes distinguishable in order to obtain the correct formula for the entropy. In order to obtain distinguishable D0 branes, we must specify some background, which completely breaks the residual symmetry of the matrix model, which is the group of permutation of $N$ objects $S_{N}$. More precisely, when we take the Matrix theory Lagrangian, which describes a system of N D0 branes, this is $0+1$ SYM theory with gauge group $\mathrm{U}(\mathrm{N})$. If we want to describe the sector of the theory with $N$ well separated D0 branes, then we must break the gauge group into $U(1)^{N}$, but the configuration is still invariant under the group of permutations $S_{N}$ which results in the quantum statistic properties of gravitons (more in [16]), But when we specify some classical background, this completely breaks the residual symmetry and we obtain a system of $N$ distinguishable particles [15].

In [15], Schwarzschild black holes were studied in the regime $S \sim N$, which simply means that the black hole consists of $\mathrm{N}$ distinguishable particles which each carry a longitudinal momentum $P_{-}=1 / R$, where $\mathrm{R}$ is the radius of the longitudinal direction in the DLCQ quantization of M theory.

An interesting proposal was made in [18], where the statistical properties of Schwarzschild black holes were obtained from first principles in Matrix theory. Because, as the authors argued, since matrix theory is a candidate for a nonperturbative description of $\mathrm{M}$ theory, it has to be possible to determine from first principles the properties of all gravitational objects included in this regime. In their paper, the thermodynamic properties were calculated from the statistical partition function of Matrix theory for a particular sector of this theory. The background was chosen to describe a collection of $\mathrm{N}$ classical D0 branes and then they calculated the partition function by integrating out off diagonal modes. Subsequently they estimated the basic thermodynamic properties of this system by the same procedure as was used in [15] for estimating mean values of characteristic parameters. After this was done, they obtained the same relation between entropy, mass and Schwarzschild radius for black hole as in classical gravity and as in the work [15] without the initial assumption that $S \sim N$. In the conclusions of [18], they suggested a similar way to count the properties of Schwarzschild black hole also in the regime $S<<N$ and they furthermore suggested that in this regime the D0 branes can form membrane-like degrees of freedom.

The regime $S<<N$ was previously investigated in the paper 24 and there it was proposed that the D0 branes would condense into $\mathrm{K}$ clusters, where $K \sim S$. In this situation these clusters must also exchange longitudinal momentum among themselves and this interaction must also be included into the effective potential between these clusters (as usual in matrix theory, it is difficult to calculate this potential directly, so the potential has to be estimated). Doing this they were able to obtain the correct value for the entropy and the mass of a Schwarzschild black hole in the regime $S<<N$.

In the present paper we will calculate the macroscopic properties of Schwarzschild black holes precisely in this regime $S<<N$ in a way similar to the one suggested in [18], so this paper should be seen as a continuation of [18]. The generalization consists in taking more complicated backgrounds with more than two objects (there are $\mathrm{K}$ extended objects), which are far away from each other. We take a background consisting of $\mathrm{K}$ clusters of D0 branes where the longitudinal momentum of the $i$-th cluster is $P_{-}=N_{i} / R$. We will compute the statistical sum, and after estimating the mean values of velocity of each object, the distance between the objects and other parameters according to [15], we obtain a value for the entropy $S \sim K$. This had to be assumed in [24], but here it emerges directly from theory. However, we cannot obtain directly from this approach the correct macroscopic quantities (mass, entropy, Schwarzschild radius) for black holes due to the fact that when we count the one loop statistical partition function, we obtain an effective potential which does not include exchange of longitudinal momentum between clusters. When we estimate this potential in the same way as in [24] we obtain correct value of mass, entropy and Schwarzschild radius 
for a Schwarzschild black hole. Certainly it would be interesting to obtain this potential directly from matrix theory, but we do not know how this could be done.

We also have to worry about the fact that membranes in Matrix theory are not stable objects unless they are infinite or wound around some non-trivial cycles. The infinite membranes are not good to construct finite size black holes from but we will show that the stable compact membranes wound on non-trivial cycles, which are described by a $3+1$ dimensional SYS theory, can be used to show that the same statements hold for Schwarzschild black holes in eight dimensions.

This paper is organized as follows. In the first part we choose a general background and expand the action for matrix theory around it giving us the action for the fluctuating fields. In the second part we evaluate the partition function to one loop order. In the third part we use our general partition function for a particular example. Namely, we consider K clusters of D0 branes and we will try to evaluate the characteristic macroscopic properties of a collection of these clusters in order to confirm the conjecture [24] that Schwarzschild black hole in the regime $S<R$ can be described as a collection of $\mathrm{K}$ clusters. In the fourth part we calculate this partition function for matrix theory compactified on a three torus and as a particular example we take a collection of $\mathrm{K}$ membranes wound around two compact directions and again we obtain the correct formula for entropy and mass of a Schwarzschild black hole.

\section{GENERAL BACKGROUND IN MATRIX THEORY}

We start with the standard action in $D L C Q M$ theory, which is $0+1 S Y M$ with gauge group $U(N)$

$$
\begin{array}{r}
S=\int d t \frac{\kappa}{2}\left[\operatorname{Tr}_{\mathrm{t}} \mathrm{X}^{\mathrm{n}} \mathrm{D}_{\mathrm{t}} \mathrm{X}^{\mathrm{n}}+\frac{\kappa}{4} \mathrm{~g}^{2} \operatorname{Tr}\left[\mathrm{X}^{\mathrm{i}}, \mathrm{X}^{\mathrm{j}}\right]\left[\mathrm{X}^{\mathrm{i}}, \mathrm{X}^{\mathrm{j}}\right]\right. \\
\left.+\frac{\kappa}{2} \operatorname{Tr}\left(\mathrm{i} \theta^{\mathrm{T}} \mathrm{D}_{\mathrm{t}} \theta+\mathrm{g} \theta^{\mathrm{T}} \gamma_{\mathrm{n}}\left[\mathrm{X}^{\mathrm{n}}, \theta\right]\right)\right] \\
D_{t} X^{n}=\partial_{t} X^{n}-i g\left[\tilde{A}, X^{m}\right], D_{t} \theta=\partial_{t} \theta-i g[\tilde{A}, \theta]
\end{array}
$$

where $\kappa=\frac{1}{R}, g=M^{3} R$ with $M$ being the Plank mass and $R$ being the radius of the compact light-like circle. Also, $\gamma^{n}$ are $S O(9)$ gamma matrices in the Majorana representation, so they are real, symmetric and obey the relation $\left\{\gamma^{m}, \gamma^{n}\right\}=2 \delta_{m n}$, and $\theta$ is thus a real 16 component spinor. Our fields also transform in the adjoint representation of the gauge group $U(N)$ so all matrices must be hermitian.

In order to evaluate the partition function, we must go to Euclidean time, so we make the transformation

$$
\tau=i t, A=-i \tilde{A}
$$

After this transformation we obtain the Euclidean form of the action

$$
\begin{array}{r}
S_{E}=\int d \tau\left[\frac{\kappa}{2} \operatorname{TrD}_{\tau} \mathrm{X}^{\mathrm{n}} \mathrm{D}_{\tau} \mathrm{X}^{\mathrm{n}}-\frac{\kappa \mathrm{g}^{2}}{4} \operatorname{Tr}\left[\mathrm{X}^{\mathrm{n}}, \mathrm{X}^{\mathrm{m}}\right]\left[\mathrm{X}^{\mathrm{n}}, \mathrm{X}^{\mathrm{m}}\right]\right. \\
\left.+\frac{\kappa}{2} \operatorname{Tr}\left(\theta^{\mathrm{T}} \mathrm{D}_{\tau} \theta-\mathrm{g} \theta^{\mathrm{T}} \gamma^{\mathrm{m}}\left[\mathrm{X}^{\mathrm{m}}, \theta\right]\right)\right]
\end{array}
$$

where $D_{\tau} X^{n}=\partial_{\tau} X^{n}-i g\left[A, X^{n}\right]$ and similarly for fermions.

We would like to evaluate the partition function, which is defined as

$$
Z(\beta)=\int[p a t h] \exp \left(-\int_{0}^{\beta} L_{E}\right)
$$

This function can be evaluated in two steps. To begin with we specify some background which is described by a classical configuration in Matrix theory and we evaluate the effective action about this background. Then, following [15], we estimate various mean values appropriate for a background describing a Schwarzschild black hole in eleven dimensions and insert them in the action to find the quantities we are interested in. After the evaluation, we find that this model do not completely describe a Schwarzschild black hole for $N>>S$ and then we explain why it is so.

We begin by computing the 1 loop effective action for an arbitrary background. To this end we divide the field from (41) into two parts: 


$$
X^{i}=\frac{1}{g} B^{i}+Y^{i}
$$

where $X^{i}$ is the classical background and $Y^{i}$ is a quantum fluctuation. We take background values of fermionic fields and ghosts fields to be zero. Because our Lagrangian is the Lagrangian of SYM theory, it is gauge invariant and we need to fix the gauge to make the calculation. We will use a gauge fixing of the form

$$
-\partial_{\tau} A+i\left[B^{n}, Y^{n}\right]=0,
$$

which means that we will also have ghosts present in the Lagrangian. After expanding the action around the background we obtain the following Lagrangian (in the following, partial derivative means derivative with respect to Euclidean time $\tau$ ):

$$
\begin{array}{r}
L_{\text {boson }}=\frac{\kappa}{2 g^{2}} \operatorname{Tr} \partial \mathrm{B}^{\mathrm{i}} \partial \mathrm{B}^{\mathrm{i}}+\frac{\kappa \mathrm{g}^{2}}{4} \operatorname{Tr}\left[\mathrm{B}^{\mathrm{n}}, \mathrm{B}^{\mathrm{m}}\right]\left[\mathrm{B}^{\mathrm{n}}, \mathrm{B}^{\mathrm{m}}\right]+ \\
+\kappa \operatorname{Tr}\left(\frac{1}{2} \partial \mathrm{Y}^{\mathrm{i}} \partial \mathrm{Y}^{\mathrm{i}}-2 \mathrm{i} \partial \mathrm{B}^{\mathrm{i}}\left[\mathrm{A}, \mathrm{Y}^{\mathrm{i}}\right]-\frac{1}{2}\left[\mathrm{~A}, \mathrm{~B}^{\mathrm{m}}\right]\left[\mathrm{A}, \mathrm{B}^{\mathrm{m}}\right]-\right. \\
-\frac{1}{2}\left[B^{n}, Y^{n}\right]\left[B^{m}, Y^{m}\right]-\frac{1}{2}\left[B^{n}, Y^{m}\right]\left[B^{n}, Y^{m}\right] \\
\left.-\frac{1}{2}\left[B^{n}, B^{m}\right]\left[Y^{n}, Y^{m}\right]--\frac{1}{2}\left[B^{n}, Y^{m}\right]\left[Y^{n}, B^{m}\right]\right) \\
L_{\text {fermi }}=\frac{\kappa}{2} \operatorname{Tr}\left(\theta^{\mathrm{T}} \partial_{\tau} \theta-\theta^{\mathrm{T}} \gamma_{\mathrm{m}}\left[\mathrm{B}_{\mathrm{n}}, \theta\right]\right) \mathrm{L}_{\text {ghost }}=\kappa \operatorname{Tr}\left(-\overline{\mathrm{c}} \partial^{2} \mathrm{c}+\overline{\mathrm{c}}\left[\mathrm{B}^{\mathrm{m}},\left[\mathrm{B}^{\mathrm{m}}, \mathrm{c}\right]\right]\right)
\end{array}
$$

where the ghost field $\bar{c}, c$ are two real and independent fermionic fields in the adjoint representation of the gauge group $U(N)$.

Now we take the background matrices in the following form

$$
B^{m}=\left(\begin{array}{cccc}
B_{1}^{m} & 0 & \ldots & 0 \\
0 & B_{2}^{m} & \ldots & 0 \\
0 & 0 & \ldots & 0 \\
0 & 0 & \ldots & B_{k}^{m}
\end{array}\right)
$$

All other background fields (including gauge field $A$ ) are chosen to be zero. The fluctuating fields have the form

$$
\begin{aligned}
A & =\left(\begin{array}{ccccc}
0 & A_{12} & A_{13} & \ldots & A_{1 K} \\
A_{12}^{\dagger} & 0 & A_{23} & \ldots & A_{2 K} \\
\ldots & \ldots & \ldots & \ldots & \ldots \\
A_{1 K}^{\dagger} & A_{2 K}^{\dagger} & \ldots & A_{K, K-1}^{\dagger} & 0
\end{array}\right) \\
Y^{m} & =\left(\begin{array}{ccccc}
0 & Y_{12}^{m} & Y_{13}^{m} & \ldots & Y_{1 K} \\
Y_{12}^{\dagger m} & 0 & Y_{23}^{m} & \ldots & Y_{2 K}^{m} \\
\ldots & \ldots & \ldots & \ldots & \ldots \\
Y_{1 K}^{\dagger m} & Y_{2 K}^{\dagger m} & \ldots & Y_{K, K-1}^{\dagger m} & 0
\end{array}\right)
\end{aligned}
$$

where $X_{m}, m=1 \ldots K$ are matrices of order $N_{m} \times N_{m}$ and $Y_{N M}, M=1 \ldots K-1, N=1 \ldots K$ are matrices of order $N_{M} \times N_{N}$. The fermionic matrix is a fluctuating matrix of the form

$$
\theta=\left(\begin{array}{ccccc}
0 & \theta_{12} & \theta_{13} & \ldots & \theta_{1 K} \\
\theta_{12}^{\dagger} & 0 & \theta_{23} & \ldots & \theta_{1 K} \\
\ldots & \ldots & \ldots & \ldots & \ldots \\
\theta_{1 K}^{\dagger} & \theta_{2 K}^{\dagger} & \ldots & \theta_{K, K-1}^{\dagger} & 0
\end{array}\right)
$$

We also have ghost fields in the form:

$$
c=\left(\begin{array}{ccccc}
0 & c_{12} & c_{13} & \ldots & c_{1 K} \\
c_{12}^{\dagger} & 0 & c_{23} & \ldots & c_{2 K} \\
\ldots & \ldots & \ldots & \ldots & \ldots \\
\ldots & \ldots & \ldots & \ldots & \ldots \\
c_{1 K}^{\dagger} & c_{2 K}^{\dagger} & \ldots & c_{K, K-1}^{\dagger} & 0
\end{array}\right)
$$


and the same matrix for $\bar{c}$, where the matrices $c$ are replaced with $\bar{c}$. These matrices with indexes $M, N$ have the same number of rows and columns as the matrix $Y_{M N}$.

This form of the matrices of the fluctuating fields is appropriate for evaluating the one loop effective action because the diagonal fluctuating fields decouple in the expansion of the Lagrangian at the quadratic level. Inserting these matrices in the Lagrangian and after straightforward but tedious algebra we obtain the following Lagrangian for the fluctuating fields:

$$
L_{b o s o n}=\kappa\left(\sum_{M<N, N=1}^{K}\left[\dot{Y}_{M N}^{\dagger a} \dot{Y}_{M N}^{a}+Y_{M N}^{\dagger a}\left(M_{M N}^{a b}\right)^{2} Y_{M N}^{b}\right]\right)
$$

where

$$
Y_{M N}^{\dagger a}=\left(Y_{M N i j}^{a}\right)^{*}
$$

is a matrix of order $N_{M} \times N_{N}$, which means that $i=1 \ldots N_{M}, j=1 \ldots N_{N}$ and the index $a$ goes from $0 \ldots 9$ where its zeroth part comes from the field $A$ and

$$
\begin{gathered}
\left(M_{M N}^{a b}\right)^{2}=M_{M N_{0}}^{a b}+M_{M N_{1}}^{a b} \\
M_{M N_{0}}^{a b}=K_{M N}^{2} \delta^{a b}, M_{M N_{1}}^{a b}=-2 i F_{M N}^{a b} \\
K_{M N}^{i}=B_{M}^{i} \otimes 1_{N_{N} \times N_{N}}-1_{N_{M} \times N_{M}} \otimes{B^{i}}_{M N}^{T} \\
F_{M N}^{i 0}=-F_{M N}^{0 i}=\dot{K}_{M N}^{i},, F_{M N}^{i j}=i\left[K_{M N}^{i}, K_{M N}^{j}\right]
\end{gathered}
$$

and by the product between vectors and matrices we mean it in a two index formulation:

$$
Y^{\dagger} C Y=\left(Y_{i j}\right)^{*} C_{i j, k l} Y_{k l}
$$

For the fermions we obtain the following result:

$$
L_{\text {fermi } i}=\kappa \sum_{M<N, N=1}^{K}\left[\theta_{M N}^{\dagger T} \dot{\theta}_{M N}-\theta_{M N}^{\dagger T} \gamma^{n} K_{M N}^{n} \theta_{M N}\right]
$$

where $\theta^{\dagger T} \theta=\left(\theta^{T}\right)_{i j}^{*} \theta_{i j}$, because the operation of transposition is related to the spinor index.

Finally we obtain the contribution from the ghosts:

$$
\begin{aligned}
L_{\text {ghost }}=\sum_{M<N, N=1}^{K}[ & -\bar{c}_{M N}^{\dagger} \partial^{2} c_{M N}+\bar{c}_{M N}^{\dagger} K_{M N}^{2} c_{M N}+ \\
& \left.+c_{M N}^{\dagger} \partial^{2} \bar{c}_{M N}-c_{M N}^{\dagger} K_{M N}^{2} \bar{c}_{M N}\right]
\end{aligned}
$$

\section{EVALUATION OF THE EFFECTIVE ACTION}

In the following we would like to calculate the one loop effective action. Because this subject is well known in the literature [20 22], we only briefly recapitulate basic facts. Firstly, because our effective action is a thermal effective action, we must specify boundary condition in the thermal direction for various fields which are in the action. We have:

$$
\Phi(0)= \pm \Phi(\beta)
$$


where the upper sign is for the bosons $\left(Y^{i}\right)$ and the lower one for the fermions $(\theta, c, \bar{c})$. We use these formulas for evaluating some integrals:

$$
\begin{aligned}
\int[Y]\left[Y^{*}\right] \exp \left(\left(Y^{a}\right)^{*} M^{a b} Y^{b}\right) & =\operatorname{det}(M)^{-1} \text { (for bosons) } \\
\int[C]\left[C^{*}\right] \exp \left(C^{*} N C\right) & =\operatorname{det}(N) \quad \text { (for fermions) }
\end{aligned}
$$

SO

$$
\exp \left(-\Gamma_{1}\right)=\prod_{M<N, N=1}^{K}(X Y Z)_{M N}
$$

where

$$
\begin{aligned}
X_{M N} & =\operatorname{det}\left(-\partial^{2}+M_{M N}\right)^{-1} \\
Y_{M N} & =\operatorname{det}\left(-\partial^{2}+K_{M N}^{2}\right)^{2} \\
Z_{M N} & =\operatorname{det}\left(\partial-\gamma^{n} K_{M N}^{n}\right)^{1}
\end{aligned}
$$

In these traces we must sum over matrix indices, Lorenz indices or spinor indices and we must integrate over $\tau$ with appropriate boundary condition. Also, the ghost determinant has exponent 2, because we have two equivalent ghosts fields.

As was explained in [19], the leading order effective action can be obtain in an adiabatic approximation

$$
\operatorname{det}\left(-\partial^{2}+\omega(\tau)^{2}\right) \sim \exp \left(\int_{0}^{\beta} \omega(t) d t\right)
$$

We must convert the determinant for fermions into a determinant which is second order in time derivatives. This can be done by using the fact that $\operatorname{det}\left(\partial+\gamma^{n} K^{n}\right)=\operatorname{det}\left(\partial-\gamma^{n} K^{n}\right)$, which gives us

$$
F^{2}=\operatorname{det}\left(\partial-\gamma^{j} K^{j}\right) \operatorname{det}\left(\partial+\gamma^{i} K^{i}\right)=\operatorname{det}\left(\partial^{2}-\frac{1}{2}\left[K^{i}, K^{j}\right] \gamma^{i j}\right)
$$

where $\gamma^{i j}=\frac{1}{2}\left[\gamma^{i}, \gamma^{j}\right]$

From this adiabatic approximation we obtain

$$
\begin{array}{r}
\Gamma_{1}=-\ln \left(\prod_{M<N, N=1}^{K}(B G F)_{M N}\right)= \\
=-\sum_{M<N, N=1}^{K}\left[\ln \left(\operatorname{det}(B)^{-1}\right)+\ln \left(\operatorname{det}(G)^{2}\right)+\ln (\operatorname{det}(F)]=\right. \\
=\sum_{M<N, N=1}^{K}\left[\int_{0}^{\beta} \operatorname{Tr}\left(\omega^{\mathrm{B}}(\mathrm{t})_{\mathrm{MN}}-\frac{1}{2} \omega^{\mathrm{F}}(\mathrm{t})_{\mathrm{MN}}-2 \omega^{\mathrm{G}}(\mathrm{t})\right)\right]
\end{array}
$$

with

$$
\begin{array}{r}
\omega^{B}(t)_{M N}^{2}=M^{2}(t)_{M N}, \omega^{F}(t)_{M N}^{2}=K_{M N}^{2} \otimes 1_{16 \times 16}-\frac{i}{2} F_{M N}^{i j} \gamma^{i j} \\
\omega^{G}(t)_{M N}^{2}=K^{2}(t)_{M N}
\end{array}
$$

In this trace we must sum over spinor or Lorenzian indices and the trace in the matrices $M_{M N i j}$ is over the $i, j$ indices. Now we must evaluate this effective action. This will be done following [21]. We must divide, the various $\Omega(t)$ into 
two parts, the first part which scales as a function of distance between two long separate object and the rest. This can be done in the following way; for bosons:

$$
\begin{aligned}
& \Omega(t)_{M N}^{a b^{2}}=M_{0 M N}^{a b}+M_{1 M N}^{a b} \\
& M(t)_{0 M N}^{a b}=K(t)_{M N}^{2} \delta^{a b}, M(t)_{1 M N}^{a b}=-2 i F_{M N}^{a b}
\end{aligned}
$$

for fermions:

$$
\begin{aligned}
& \Omega(t)_{M N}^{2}=M(t)_{0 M N}+M(t)_{1 M N} \\
& \quad M(t)_{0 M N}=K(t)_{M N}^{2} \otimes 1_{16 \times 16}, M(t)_{1 M N}=\partial K(t)_{M N}^{n} \gamma^{n}-\frac{1}{2} F_{M N}^{i j} \gamma^{i j}
\end{aligned}
$$

We must go over to the proper time representation :

$$
\operatorname{Tr} \sqrt{\mathrm{M}(\mathrm{t})_{0 \mathrm{MN}}+\mathrm{M}(\mathrm{t})_{1 \mathrm{MN}}}=-\frac{1}{2 \sqrt{\pi}} \operatorname{Tr} \int_{0}^{\infty} \frac{\mathrm{d} \tau}{\tau^{\frac{3}{2}}} \exp \left(-\tau\left(\mathrm{M}(\mathrm{t})_{0}+\mathrm{M}(\mathrm{t})_{1}\right)\right)
$$

The integral can now be evaluated by the help of a Dyson perturbative series. Because we know, that $M_{1}$ is much smaller than $M_{0}$, we can take the latter as a perturbative term, and the former as a background around which we expand. It corresponds to the standard Dyson formula free Hamiltonian. We take (in the following we will not write out the dependence of these terms on $t$, because in the proper time representation these terms are constant. We also will not write various indices, because these are not important in what follows)

$$
\begin{gathered}
U(s)=\exp ^{-s\left(M_{0}+N_{1}\right)}, U(s)_{0}=\exp ^{-s M_{0}} \\
V(s)=U(-s)_{0} U(s), \frac{d V(s)}{d s}=-M(s)_{1} V(s) M(s)_{1}=\exp ^{i s M_{0}} M_{1} \exp ^{-i s M_{0}} \\
V(s)=1-\int_{0}^{s} M\left(s_{1}\right)_{1} d s_{1}+\int_{0}^{s} M\left(s_{2}\right) \int_{0}^{s_{2}} M\left(s_{1}\right)_{1} d s_{1} d s_{2} \ldots \\
U(s)=U(s)_{0} V(s), \operatorname{Tr} \sqrt{\mathrm{M}_{0}+\mathrm{M}_{1}}=-\frac{1}{2 \sqrt{\pi}} \operatorname{Tr} \int_{0}^{\infty} \frac{\mathrm{ds}}{\mathrm{s}^{\frac{3}{2}}} \mathrm{U}(\mathrm{s})
\end{gathered}
$$

Zero order:

$$
\operatorname{Tr}_{\mathrm{L}}\left(1_{10 \times 10}\right)-\frac{1}{2} \operatorname{Tr}_{\mathrm{L}}\left(1_{16 \times 16}\right)-2=0
$$

where in evaluating we divide the trace into two parts, one over the Lorenz indices and the other over the matrix indices. In the following we will not write indices of type $M N$, because as we can see from (29), the effective action is linear in indexes $M, N$ so that we can simply calculate for one matrix with index $M N$ and then sum over $M, N$. We see that to zeroth order these contributions are zero. To first order, we have contributions from boson and fermion respectively:

$$
\operatorname{Tr}_{L} M(s)_{1 B}-\operatorname{Tr}_{L} M(s)_{1 F}=-2 i F^{a a}+\partial K^{n} \operatorname{Tr} \gamma^{n}-\frac{i}{2} F^{i j} \otimes \operatorname{Tr} \gamma^{i j}=0
$$

This is due to the basic properties of the gamma matrices $\left(\operatorname{Tr} \gamma^{\mathrm{n}}=0\right)$ and the antisymmetry of tensor $F^{a b}$.

To second order, we obtain the following term in the effective action:

$$
-\frac{1}{2 \sqrt{2}} \operatorname{Tr}_{\mathrm{G}} \int_{0}^{\infty} \frac{\mathrm{ds}}{\mathrm{s}^{\frac{3}{2}}} \exp ^{-\mathrm{sM}_{0}} \operatorname{Tr}_{\mathrm{L}}\left(\int_{0}^{\mathrm{s}} \mathrm{ds}_{1} \mathrm{M}\left(\mathrm{s}_{1}\right) \int_{0}^{\mathrm{s}_{1}} \mathrm{ds}_{2} \mathrm{M}\left(\mathrm{s}_{2}\right)\right)
$$

The bosonic term has the form: 


$$
\operatorname{Tr}_{\mathrm{L}}\left(\mathrm{M}\left(\mathrm{s}_{1}\right)_{1} \mathrm{M}\left(\mathrm{s}_{2}\right)_{1}\right)=8 \dot{\mathrm{K}}\left(\mathrm{s}_{1}\right) \dot{\mathrm{K}}\left(\mathrm{s}_{2}\right)-4 \mathrm{~F}^{\mathrm{ij}}\left(\mathrm{s}_{1}\right) \mathrm{F}^{\mathrm{ij}}\left(\mathrm{s}_{2}\right)
$$

and the fermionic term has the form:

$$
\begin{array}{r}
\operatorname{Tr}_{\mathrm{L}}\left(\mathrm{M}\left(\mathrm{s}_{1}\right)_{1} \mathrm{M}\left(\mathrm{s}_{2}\right)_{2}\right)= \\
=\left(\dot{K}^{n}\left(s_{1}\right) \gamma^{n}-\frac{i}{2} F^{i j}\left(s_{1}\right) \gamma^{i j}\right)\left(\dot{K}^{m}\left(s_{2}\right) \gamma^{m}-\frac{i}{2} F^{k l}\left(s_{2}\right) \gamma^{k l}\right)= \\
=\dot{K}^{n}\left(s_{1}\right) \dot{K}^{m}\left(s_{2}\right) \delta^{m n}-\frac{1}{4} F^{i j}\left(s_{1}\right) F^{k l}\left(s_{2}\right) \operatorname{Tr}\left(\gamma^{\mathrm{ij}} \gamma^{\mathrm{kl}}\right)= \\
=16 \dot{K}\left(s_{1}\right) \dot{K}\left(s_{2}\right)-8 F^{i j}\left(s_{1}\right) F^{j i}\left(s_{2}\right)
\end{array}
$$

so fermionic and bosonic contribution cancel each other. To third order we obtain:

$$
\frac{1}{\sqrt{2} \sqrt{\pi}} \int_{0}^{\infty} \frac{d s}{s^{\frac{3}{2}}} \exp ^{-s M_{0}} \int_{0}^{s} d s_{1} M\left(s_{1}\right)_{1} \int_{0}^{s_{1}} d s_{2} M\left(s_{2}\right)_{1} \int_{0}^{s_{2}} d s_{3} M\left(s_{3}\right) d s_{3}
$$

which for bosons means:

$$
\begin{array}{r}
\operatorname{Tr}_{\mathrm{L}}\left(\mathrm{M}\left(\mathrm{s}_{1}\right)_{1} \mathrm{M}\left(\mathrm{s}_{2}\right)_{1} \mathrm{M}\left(\mathrm{s}_{3}\right)_{1}\right)= \\
=8 i\left(-\dot{K}^{i}\left(s_{1}\right) F_{i j}\left(s_{2}\right) \dot{K}^{j}\left(s_{3}\right)-F^{i j} \dot{K}^{j}\left(s_{2}\right) \dot{K}^{i}\left(s_{3}\right)-\right. \\
\left.-\dot{K}^{j}\left(s_{1}\right) \dot{K}^{i}\left(s_{2}\right) F^{i j}\left(s_{3}\right)+F^{i j}\left(s_{1}\right) F^{j k}\left(s_{2}\right) F^{k i}\left(s_{3}\right)\right)
\end{array}
$$

and for fermions:

$$
\begin{array}{r}
\operatorname{Tr}_{\mathrm{L}}\left(\mathrm{M}\left(\mathrm{s}_{1}\right)_{1} \mathrm{M}\left(\mathrm{s}_{2}\right)_{1} \mathrm{M}\left(\mathrm{s}_{3}\right)_{1}\right)=\operatorname{Tr}_{\mathrm{L}}\left(\dot{\mathrm{K}}^{\mathrm{n}} \gamma^{\mathrm{n}}-\frac{\mathrm{i}}{2} \mathrm{~F}^{\mathrm{ij}} \gamma^{\mathrm{ij}}\right)\left(\mathrm{s}_{1}\right) \times \\
\times\left(\dot{K}^{m} \gamma^{m}-\frac{i}{2} F^{k l} \gamma^{k l}\right)\left(s_{2}\right)\left(\dot{K}^{p} \gamma^{p}-\frac{i}{2} F^{o p} \gamma^{o p}\right)\left(s_{3}\right)=
\end{array}
$$

where the trace is now over spinor indices. After some algebra we obtain the following result for the fermions:

$$
=\left(-16 i F^{i j} F^{j k} F^{k i}-16 i \dot{K}^{i} F^{i j} \dot{K}^{j}-16 i F^{i j} \dot{K}^{i} \dot{K}^{j}+16 i F^{i j} F^{j k} F^{k i}\right)\left(s_{1}\right)\left(s_{2}\right)\left(s_{3}\right)
$$

so again, to third order, fermionic and bosonic term cancel each other. The first nontrivial term we obtain at fourth order, so when we take the leading terms of $M_{1}(s)$, which is independent of $s$, we obtain the following result

$$
\begin{array}{r}
\operatorname{Tr}_{\mathrm{L} \text { boson }}\left(\mathrm{M}\left(\mathrm{s}_{1}\right)_{1} \mathrm{M}\left(\mathrm{s}_{2}\right)_{1} \mathrm{M}\left(\mathrm{s}_{3}\right)_{1} \mathrm{M}\left(\mathrm{s}_{4}\right)_{1}\right)_{\mathrm{MN}} \\
-\frac{1}{2} \operatorname{Tr}_{\mathrm{L} \text { fermi }}\left(\mathrm{M}\left(\mathrm{s}_{1}\right)_{1} \mathrm{M}\left(\mathrm{s}_{2}\right)_{1} \mathrm{M}\left(\mathrm{s}_{3}\right)_{1} \mathrm{M}\left(\mathrm{s}_{4}\right)_{1}\right)_{\mathrm{MN}}= \\
=F_{M N}=\operatorname{Str}\left(24 \mathrm{~F}_{\mathrm{oi}} \mathrm{F}_{\mathrm{oi}} \mathrm{F}_{\mathrm{oj}} \mathrm{F}_{\mathrm{oj}}-24 \mathrm{~F}_{0 \mathrm{i}} \mathrm{F}_{\mathrm{oi}} \mathrm{F}_{\mathrm{jk}} \mathrm{F}_{\mathrm{jk}}-\right. \\
\left.-96 F_{o i} F_{o j} F_{i k} F_{k j}+24 F_{i j} F_{j k} F_{k l} F_{l i}-6 F_{i j} F_{i j} F_{k l} F_{k l}\right)
\end{array}
$$

where Str, meaning the symmetric trace, is defined as an average over all ordering in indices $i j$ and to leading order we may replace $M_{0}$ in the exponential function in our Dyson series by the term $r_{M N}$ so after a simple integration, and because the trace is independent to leading order in $s$ we obtain the final form for the effective potential:

$$
\Gamma_{1}=\frac{-5}{128} \sum_{M<N, N=1}^{K} \int_{0}^{\beta} \frac{d t}{r_{M N}^{7}} \operatorname{StrF}(\mathrm{t})_{\mathrm{MN}}
$$

This is the final result, which can be used for studying the thermodynamic properties of Schwarzschild black holes. 


\section{SCHWARZSCHILD BLACK HOLES}

We will follow [15] in supposing that we have some bound state corresponding to some classical object in matrix theory which move in any bounded region in space time and then we will try to evaluate the thermodynamic characteristics of this system. We will take our background in the form of $K$ clusters of $D 0$ branes which are far away from each other and we will suppose that their quantum mechanical properties are not important for obtaining the leading order result. So we will take the background in the following form:

$$
X^{m}=\left(\begin{array}{ccc}
\left(v_{1}^{m} \beta+r_{1}^{m}\right) \otimes 1_{N_{1} \times N_{1}} & 0 & \\
0 & \left(v_{2}^{m} \beta+r_{2}^{m}\right) \otimes 1_{N_{2} \times N_{2}} & 0 \\
0 & \cdots & \left(v_{K}^{m} \beta+r_{K}^{m}\right) \otimes 1_{N_{K} \times N_{K}}
\end{array}\right)
$$

where $r_{i}^{m}$ describes position of the cluster in time $\beta=0$ and where $v_{i}^{m}$ is the velocity of the cluster and $m=1 \ldots 9$. By choosing a background of this form we do not care about the proper structure of the bound state (cluster) of these particles. Inserting these background matrices into the effective action we obtain:

$$
\begin{gathered}
\Gamma_{0}=\beta \sum_{M=1}^{K} \frac{1}{2 R} N_{M} v_{M}^{2} \\
\Gamma_{1}=-\frac{15}{16} \sum_{M<N, N=1}^{K} \int_{0}^{\beta} d t \frac{N_{N} N_{M} v_{M N}^{4}}{\left(v_{M N}^{2} t^{2}+r_{M N}^{2}\right)^{\frac{7}{2}}}
\end{gathered}
$$

Now we must make a scale transformation, because this effective action has been evaluated for background matrices $B^{n}$ and these are related to the physical coordinates and physical velocities through the following transformations:

$$
B^{i}=g X^{i}, g=M_{p}^{3} R, v \rightarrow g v, \Gamma_{1} \rightarrow \frac{1}{g^{3}} \Gamma_{1}
$$

After this transformation we obtain as a final result:

$$
\Gamma=\Gamma_{0}+\Gamma_{1}=\beta \sum_{N=1}^{K} \frac{N_{N} v_{N}^{2}}{2 R}-\frac{15 l_{P}^{9}}{16 R^{3}} \sum_{M<N, N=1}^{K} \int_{0}^{\beta} d t \frac{N_{M} N_{M} v_{M N}^{4}}{\left(v_{M N}^{2} t^{2}+r_{M N}^{2}\right)^{\frac{7}{2}}}
$$

Now we would like to evaluate some thermodynamic properties for this ensemble. We will suppose that these clusters live in some bounded region in space-time and that the radius of this region is $R_{s}$. Since these clusters must live in this region, their thermal average value must obey the following conditions:

$$
\left\langle\left|r_{M N}\right|\right\rangle \sim\left\langle\left|v_{M N}\right|\right\rangle \beta \sim\left\langle\left|v_{M}\right|\right\rangle \beta \sim R_{s}
$$

where $\beta$ is the inverse temperature of the black hole. The cluster must obey the Heisenberg uncertainty principle, which in our situation says

$$
\frac{\left\langle N_{M}\right\rangle}{R}\left\langle\left|v_{M}\right|\right\rangle R_{s} \sim 1
$$

because the momentum of the cluster (as can be derived from the Lagrangian, using Noether's theorem, since our Lagrangian is invariant under translation) is

$$
P_{M}^{i}=\frac{N_{M} v_{M}}{R}
$$

We can also suppose that all clusters have on the average the same value of longitudinal momentum $P_{-}$, which tells us that

$$
\left\langle N_{M}\right\rangle \sim \frac{N}{K}
$$

When we combine all these facts, we obtain following average values: 


$$
\left\langle\left|v_{M}\right|\right\rangle \sim\left\langle\left|v_{M N}\right|\right\rangle \sim \frac{R K}{N R_{s}}, \beta \sim \frac{R_{s}^{2} N}{R K}
$$

Now, since these average values have been estimated, we can start to count the statistical properties of this ensemble. We have

$$
\begin{gathered}
Z(\beta)=\sum_{\text {overparametrs }} \exp ^{-\left(\Gamma_{0}+\Gamma_{1}\right)}=\left\langle\exp ^{-\left(\Gamma_{0}+\Gamma_{1}\right)(\beta)}\right\rangle \\
\langle E\rangle=-\frac{\partial \ln Z(\beta)}{\partial \beta}=\left\langle\frac{\partial\left(\Gamma_{0}+\Gamma_{1}\right)}{\partial \beta}\right\rangle
\end{gathered}
$$

when we apply our average values, we obtain

$$
\langle E\rangle \sim \frac{R K^{2} N}{2 R_{s}^{2} N^{2}}-\frac{15 G_{11} R K^{4}}{32 N^{2} R_{s}^{11}}
$$

The Helmholtz free energy is given by

$$
\begin{array}{r}
F=-\frac{\ln Z(\beta)}{\beta}= \\
\left\langle\sum_{N=1}^{K} \frac{N_{N} v_{N}^{2}}{2 R}-\frac{15 G_{11}}{16 R^{3}} \sum_{M<N, N=1}^{K} \frac{1}{\beta} \int_{0}^{\beta} d t \frac{N_{N} N_{M} v_{M N}^{4}}{\left(v_{M N}^{2} t^{2}+r_{M N}^{2}\right)^{\frac{7}{2}}}\right\rangle
\end{array}
$$

From this free energy we can obtain the entropy:

$$
\begin{aligned}
S=\beta^{2} \frac{\partial F(\beta)}{\partial \beta}= & \left\langle\frac{15 G_{11}}{16 R^{3}} \sum_{M<N, N=1}^{K} \int_{0}^{\beta} \frac{N_{N} N_{M} v_{N M}^{4}}{\left(v_{M N}^{2} t^{2}+r_{M N}^{2}\right)^{\frac{7}{2}}}-\right. \\
& \left.-\beta \frac{15 G_{11}}{16 R^{3}} \sum_{M<N, N=1}^{K} \frac{N_{N} N_{M}}{\left(v_{M N}^{2} \beta^{2}+r_{M N}^{2}\right)^{\frac{7}{2}}}\right\rangle
\end{aligned}
$$

Inserting our average values into the previous term, we obtain the entropy in the following form

$$
S \sim \frac{G_{11} K^{3} N^{2}}{R_{s}^{9} N^{3}}
$$

In order to obtain the value for the radius $R_{s}$, we apply the virial theorem, which has the form

$$
\begin{array}{r}
\left\langle E_{\text {kin }}\right\rangle \sim\left\langle E_{\text {pot }}\right\rangle \rightarrow \\
\rightarrow \frac{R K^{2} N}{R_{s}^{2} N^{2}} \sim \frac{G_{11} R K^{3}}{N^{2} R_{s}^{11}} \rightarrow R_{s} \sim\left(\frac{K^{2} G_{11}}{N}\right)^{\frac{1}{9}}
\end{array}
$$

When we use the previous result for estimating the entropy, we obtain

$$
S \sim K<<N
$$

So our result confirms the conjecture of [24], that Schwarzschild black holes in region $S<<N$ can be described as a bound state of $K$ clusters, but this is not the end of the story.

When we insert (64) into 60 we obtain

$$
E \sim R G_{11}^{-\frac{2}{9}} K^{\frac{14}{9}} N^{-\frac{7}{9}}
$$


and when we use the relation between mass and light-cone energy, we obtain a final result for the mass of a Schwarzschild black hole:

$$
M^{2}=2 P_{-} E \rightarrow M \sim\left(G_{11}^{-1} N\right)^{\frac{1}{9}} K^{\frac{7}{9}}
$$

which certainly is not the correct result, since mass is a function of the number $N$, but mass is a macroscopic quantity which cannot depend on microscopic parameters. The reason why we cannot obtain a correct result lies in our ignorance of the process of longitudinal momentum exchange. But this is difficult problem in matrix theory, which cannot be resolved with our simple result. But if we follow [24 and estimate the interaction term in the way that was used in that paper we obtain

$$
E_{\text {pot }} \sim-\frac{N}{K}\left\langle\Gamma_{1}\right\rangle
$$

so the average value of the energy is

$$
\langle E\rangle \sim \frac{R K^{2} N}{2 R_{s}^{2} N^{2}}-\frac{15 N G_{11} R K^{4}}{32 K N^{2} R_{s}^{11}}
$$

and when we apply virial theorem to (69), we obtain

$$
R_{s} \sim\left(G_{11} K\right)^{\frac{1}{9}}
$$

When we count entropy in the same way as before, we obtain

$$
S \sim \frac{G_{11} N^{3} K^{3}}{R_{s}^{9} K N^{3}} \sim \frac{G_{11} K^{2}}{R_{s}^{9}} \sim K
$$

and the energy

$$
\begin{array}{r}
\langle E\rangle \sim \frac{R K^{2} N}{2 R_{s}^{2} N^{2}}-\frac{15 G_{11} N K^{4} R}{32 K N^{2} R_{s}^{11}} \sim \\
\sim \frac{R}{N}\left(\frac{K^{8}}{G_{11}}\right)^{\frac{2}{9}}
\end{array}
$$

and the mass of Schwarzschild black hole becomes

$$
M=\sqrt{\frac{N}{R} E} \sim\left(\frac{K^{8}}{G_{11}}\right)^{\frac{2}{9}}
$$

and finally, when we insert (73) into (70), we obtain

$$
R_{s} \sim\left(G_{11} M\right)^{\frac{1}{8}}
$$

which is the correct result for the Schwarzschild radius as a function of the mass of the black hole. Finally, we will determine the temperature of the Schwarzschild black hole. To begin with we write the transformation rule in DLCQ for a transformation in the longitudinal direction:

$$
\left[M_{+-}, P_{+}\right]=i P_{+},\left[M_{+-}, P_{-}\right]=-i P_{-}
$$

which means, that these two quantities scale with relatively inverse coefficient under this transformation. And because temperature transforms as an energy, we have:

$$
\begin{array}{r}
P_{-}=k P_{0-} \rightarrow k=\frac{P_{-}}{P_{0-}},\left(P_{-}=\frac{N}{R}, P_{0-}=\frac{M}{\sqrt{2}}\right) \\
\frac{1}{\beta}=k^{-1} T_{0} \rightarrow T_{0}=\frac{\sqrt{2} N}{R M \beta} \sim \frac{1}{R_{s}}
\end{array}
$$

again this is a correct result. 


\section{OBJECTS IN MATRIX THEORY ON A TORUS}

So far we have described only flat eleven dimensional matrix theory and general configuration matrix objects in this theory. We would like to confirm the conjecture of [18], that the Schwarzschild black hole in the regime $S<<N$ can be described as a collection of S membranes. However, there is a problem. The previous effective action is certainly valid formally, but cannot describe extended objects in matrix theory for finite $\mathrm{N}$. This is because these objects are described by commutator $F^{i j}=i\left[K^{i}, K^{j}\right]$ terms, of which the trace is certainly zero for finite matrices. These extended objects should therefore be described by infinite matrices, but this is not the end of the story. Even classically, membranes are stable only if they are infinite, or if they are living on some compact manifold. Finite membranes in flat space-time will eventually collapse into a point in the classical case, but when their size will be of the order of the Planck scale, the quantum mechanical properties will be important so then we must study the whole system as a quantum mechanical system which is described by a special $0+1 \mathrm{SYM}$ with gauge group $U(N)$ and this is a rather difficult problem. On the other hand, classical infinite membranes cannot serve as a model for Schwarzschild black hole. In order to describe a statistical ensemble of stable extended objects (for example membranes), we must take these extended objects as being infinite or we must wind them on some compact dimension. In order to describe a membrane, which is finite, we must take Matrix theory on a compact manifold, and the simplest way is to compactify Matrix theory on a p-torus, which by the standard prescription 23] is the same as SYM on the dual p-torus. In the following we will study membranes which are wound over a 3-torus so Matrix theory is described by $3+1$ SYM with gauge group $U(N)$, for a system with total longitudinal momentum $P_{-}=\frac{N}{R}$. The bosonic part of this Lagrangian has the form:

$$
L=\frac{1}{g^{2}} \int d^{p} \tilde{x} \operatorname{Tr}\left(-\frac{1}{4} \mathrm{~F}_{\mu \nu} \mathrm{F}^{\mu \nu}+\frac{\left(\mathrm{D}_{\mu} \phi\right)^{2}}{2}+\left[\phi^{\mathrm{i}}, \phi^{\mathrm{j}}\right]^{2}\right)
$$

where the integration is over the dual 3 torus of size (for simplicity all compact dimensions have the same size)

$\Sigma=\frac{2 \pi l_{p}^{3}}{R L}$. Here $R$ is the radius of the longitudinal direction and $L$ is the radius of the compact dimension of the original torus. Greek indices run over $0 \ldots 3$ and over $i, j=4 \ldots 9$. In the Yang-Mills theory, the coupling constant is

$$
g^{2}=\frac{l_{p}^{3}}{L^{3}}
$$

We would like to show how the action for the membrane arise from the previous Lagrangian since the generalization for more membranes is straightforward. (It simply consists in breaking gauge group $U(N)$ into $U\left(N_{1}\right) \times U\left(N_{2}\right) \times \ldots U\left(N_{K}\right)$, where each subgroup describes one particular membrane.)

We would like to describe a membrane moving in the fourth direction, and which is wound in the first and second direction. In order to describe this membrane we take a classical background in the following way. We suppose that $\phi$ are position independent and that there are no Wilson lines $\oint A_{\mu} d x^{\mu}=0$. Then the kinetic term for the scalar field reduces to the form

$$
\frac{1}{g^{2}} \tilde{V} \dot{\phi}^{2}
$$

where $\tilde{V}$ is the volume of the dual torus which is related to the volume of the original torus $\mathrm{V}$ through the standard form $\tilde{V}=\frac{l_{p}^{9}(2 \pi)^{3}}{R^{3} V}$. We must also relate this scalar field with the physical momentum of the membrane in the fourth direction. This also means that the only nonzero field is $\phi_{4}$, all the others are zero. We take this field to be of the form :

$$
\dot{\phi}^{i}=K^{i} \otimes 1_{N \times N}
$$

The unknown constant $\mathrm{K}$ can be determined from this condition. The action with (77) is invariant under the transformation:

$$
\phi^{\prime}{ }^{i}=\phi^{i}+b^{i} \otimes 1_{N \times N}
$$

where $b^{i}$ is an arbitrary constant. This invariance is nothing but Poincare translation symmetry in the transversal space. By using standard Noether methods we obtain conserved quantities which are just the moment in the transverse dimensions:

$$
P^{i}=\frac{1}{g^{2}} \int \operatorname{Tr} \dot{\phi}^{\mathrm{i}} \mathrm{dx}=\frac{1}{\mathrm{~g}^{2}} \tilde{\mathrm{V}} \operatorname{Tr} \dot{\phi^{\mathrm{i}}}
$$


and finally, when we insert our background ansatz (79) into (81) and using (78), we obtain

$$
P=\frac{K N L^{3} l_{p}^{9}(2 \pi)^{3}}{l_{p}^{3} R^{3} L^{3}} \Rightarrow K=P\left(\frac{R}{l_{p}^{3}(2 \pi)^{3}}\right)^{2} \frac{1}{P_{-}}
$$

As a check, we may insert (79) into the Hamiltonian, which has the form

$$
H=\frac{1}{g^{2}} \int \operatorname{Tr}\left(\frac{1}{4} \mathrm{~F}_{\mu \nu} \mathrm{F}^{\mu \nu}+\frac{1}{2} \dot{\phi}^{2}\right)
$$

and if we for the moment leave out the gauge field term, we obtain

$$
H=\frac{1}{g^{2}} \operatorname{Tr} \dot{\phi}^{2} \tilde{\mathrm{V}}=\frac{\mathrm{P}^{2} \mathrm{R}}{\mathrm{N}}=\frac{\mathrm{P}^{2}}{\mathrm{P}_{-}}
$$

which is the correct result.

In order to describe a membrane, we must also have a non-vanishing value of magnetic flux and when the membrane is wound on the first and second direction, this flux is

$$
\frac{1}{2 \pi} \int \operatorname{TrF}_{12} \mathrm{dx}^{1} \mathrm{dx} \mathrm{x}^{2}=\mathrm{n}
$$

where the integral is over the first and second dimension and $n$ is an integer. Again, when our background configuration is independent of the space coordinates, we obtain the following value for $F_{12}$

$$
F_{12}=\frac{n}{N} \frac{2 \pi}{\Sigma^{2}}=\frac{n}{N} \frac{R^{2} L^{2}}{2 \pi l_{p}^{6}}
$$

and when we insert the previous result into 83), we obtain

$$
\frac{1}{2 g^{2}} F_{12}^{2} \tilde{V}=\frac{R}{2 N} n^{2}(2 \pi) \frac{L^{4}}{l_{p}^{6}}=\frac{2 \pi M^{2}}{2 P_{-}}
$$

from which we obtain the correct mass of a membrane which is $n$ times wound around the first and second direction.

$$
M=\frac{L^{2} n}{l_{p}^{3}}
$$

Now when we take the background in the following form:

$$
F_{12}=\left(\begin{array}{cccc}
F^{1} \otimes 1_{N_{1} \times N_{1}} & 0 & \ldots & 0 \\
0 & F^{2} \otimes 1_{N_{2} \times N_{2}} & \ldots & 0 \\
\ldots & \ldots & \ldots & \ldots \\
0 & \ldots & \ldots & F^{K} \otimes 1_{N_{K} \times N_{K}}
\end{array}\right)
$$

where we have

$$
\frac{1}{2 \pi} \int \operatorname{TrF}_{12}^{\mathrm{i}}=\frac{1}{2 \pi} \tilde{\mathrm{V}} \mathrm{NF}^{\mathrm{i}}=\mathrm{n}^{\mathrm{i}}
$$

and

$$
\phi^{4}=\left(\begin{array}{cccc}
k^{1} t \otimes 1_{N_{1} \times N_{1}} & 0 & \ldots & 0 \\
0 & k^{2} t \otimes 1_{N_{2} \times N_{2}} & \ldots & 0 \\
\ldots & \ldots & \ldots & \ldots \\
0 & \ldots & \ldots & k^{K} t \otimes 1_{N_{K} \times N_{K}}
\end{array}\right)
$$

and insert it into the effective action, we obtain the effective action for $\mathrm{K}$ membranes in the form:

$$
\Gamma_{0}=\beta \sum_{i=1}^{K}\left(\frac{P_{i}^{2}}{2 P_{i-}}+\frac{2 \pi M_{i}^{2}}{2 P_{i-}}\right)
$$


We express this effective action in momentum variables because this is more appropriate for compactification (we can take the momentum to be finite), but from (88) we see that in the limit $L \rightarrow 0$, mass goes to zero, so that the membrane looks like it lives in a non-compact dimension as a D0 brane.

Now we may start to analyze the effective potential between the K membranes described by the previous background. This potential was obtained in a previous work [20], which in our case, $3+1 S Y M$, takes the form:

$$
\Gamma_{1}=-\sum_{M<N, N=1}^{K} \frac{a}{r_{M N}^{4}} \int_{0}^{\beta} d \tilde{x} \operatorname{Str}\left(\mathrm{F}_{\mathrm{MN}}\right)
$$

where the minus sign is due to the fact that we are calculating the finite temperature effective potential which means that we are working in Euclidean signature and

$$
\begin{array}{r}
F_{M N}=\left(24 F_{o i} F_{o i} F_{o j} F_{o j}-24 F_{o i} F_{o i} F_{j k} F_{j k}\right. \\
-96 F_{o i} F_{o j} F_{i k} F_{k j}+24 F_{i j} F_{j k} F_{k l} F_{l i} \\
\left.-6 F_{i j} F_{i j} F_{k l} F_{k l}\right)_{M N}
\end{array}
$$

For our background we obtain these nonzero terms

$$
\begin{array}{r}
F_{12 M N}=F_{12 M} \otimes 1_{N \times N}+1_{M \times M} \otimes F_{12 N}^{T} \\
F_{04 M N}=\dot{\phi}_{4 M} \otimes 1_{N \times N}-1_{M \times M} \otimes \dot{\phi}_{4 N}
\end{array}
$$

When we insert this background into (93) and using

$$
\operatorname{Tr}\left(\mathrm{A}_{\mathrm{M}} \otimes \mathrm{A}_{\mathrm{N}} \mathrm{B}_{\mathrm{M}} \otimes \mathrm{B}_{\mathrm{N}} \ldots\right)=\operatorname{Tr}\left(\mathrm{A}_{\mathrm{M}} \mathrm{B}_{\mathrm{M}} \ldots\right) \operatorname{Tr}\left(\mathrm{A}_{\mathrm{N}} \mathrm{B}_{\mathrm{N}} \ldots\right)
$$

and using the fact that for a commuting background (such as in our case) Str is the same as an ordinary trace, we obtain

$$
\begin{array}{r}
\Gamma_{1}=-\frac{A G_{8}}{R} \sum_{M<N, N=1}^{N} \int_{0}^{\beta} d t \frac{P_{-M} P_{-N}}{r_{M N}^{4}} \times \\
\times\left[\left(\frac{P_{M}}{P_{-M}}-\frac{P_{N}}{P_{-N}}\right)^{2}-\frac{L^{2}}{(2 \pi)^{2}}\left(\frac{n_{N}}{N_{N}}+\frac{n_{M}}{N_{M}}\right)^{2}\right]^{2}
\end{array}
$$

where $A$ is a numerical constant, which is not important for our purposes $G_{8}=\frac{l_{p}^{9}}{L^{3}}$, and the meaning of the other symbols was explained in the previous part where we considered one simple membrane. Again we see that in the limit $L \rightarrow 0$ the last term in the effective potential is zero, so again this effective action reduces to the effective action of $\mathrm{K}$ D0 branes in eight dimensions:

$$
\begin{array}{r}
\Gamma=\Gamma_{0}+\Gamma_{1}= \\
\beta \sum_{i=1}^{K} \frac{P_{i}^{2}}{P_{-i}}-\frac{A G_{8}}{R} \int_{0}^{\beta} d t \frac{P_{-M} P_{+N}}{r(t)_{M N}^{4}}\left(\frac{P_{M}}{P_{-M}}-\frac{P_{N}}{P_{-N}}\right)^{4}
\end{array}
$$

We see that again this configuration describe an ensemble of K D0 particles, so that the analysis will be the same as in the previous case or as in [24], so that we will not repeat this analysis there.

\section{CONCLUSION}

In the previous parts we have done some simple calculations in Matrix theory. The basic goals of this paper was to confirm the conjecture in [18], that a Schwarzschild black hole in the regime $N>S$ can be described as a 
collection of $\mathrm{K}$ membranes which each has a longitudinal momentum $P_{-} \sim N / K R$. This goal was achieved in the sense that we managed to derive, from first principles, that the entropy is proportional to the number of membranes. By estimating the correct potential energy between these clusters we also managed to derive the correct values for the other parameters of the black hole. We also showed that the same results hold when we use membrane constituents that are stable against collapse, namely, which are wound around some compact manifold.

Clearly, it would be of great interest to also calculate the potential between the clusters from first principles. This, however, is a process that involves longitudinal momentum transfer which is a notoriously difficult problem in matrix theory so we will leave this problem for the future.

\section{ACKNOWLEDGEMENTS}

I would like to thank Rikard von Unge and Zdeňek Kopecký for helpful discussions.

[1] T. Banks , W. Fischer, S. Schenker and L. Susskind, "M Theory As A Matrix Model: Conjecture", Phys. Rev. D55, 5112-5128,(1997), hep-th/

[2] K. Becker and M. Becker, "Two Loop Test of M(atrix) Theory", Nucl. Phys. B 506,48-60, (1997) hep-th/9705091

[3] Y. Okawa and T. Yoneya, "Multi Body Interactions of D-Particles in Supergravity and Matrix Theory", Nucl. Phys. B538, 67-99,(1999), hep-th/9806108

[4] W. Taylor and M. Van Raamsdonk, "Three- graviton scattering in Matrix theory revised", Phys.Lett. B438, 248-254, (1998), hep-th/9806066

[5] L. Susskind, W. Fischer, E. Halyo and A. Rajaraman, "The Incredible Shrinking Torus", Nucl. Phys. B501, 409-426, (1997)hep-th/9703102

[6] N. A. Obers, B. Pioline and E. Rabinovici, "M- Theory and U- duality on $T^{d}$ with Gauge Backgrounds", Nucl. Phys. B525, (163-181), hep-th/9712084

[7] A. Sen, "D0 Branes on $T^{n}$ and Matrix Theory", Adv. Theor. Math. Phys. 2, 51-59, (1998), hep-th/9709220

[8] N. Seiberg, "Why is the Matrix Model Correct? ", Phys. Rev. Lett. 79, 3577-3580,(1997), hep-th/9710009

[9] N. Seiberg, "Matrix Description of M theory on $T^{5}$ and $T^{5} / Z_{2}$ ", Phys. Lett. B408, 98-104, (1997), hep-th/9705221

[10] L. Motl, "Proposals on nonperturbative superstring interaction", hep-th/9701025

[11] T. Banks and N. Seiberg, "Strings from Matrices" Nucl. Phys. B497, 41-55, (1997) hep-th/9702187

[12] R. Dijkgraaf, E. Verlinde and H. Verlinde, "Matrix String Theory" Nucl. Phys. B500, 43-61, (1997), hep-th/9703030

[13] , T. Banks and L. Motl, "Heterotic Strings from Matrices", JHEP 9712, 004, (1997), hep-th/9703218

[14] M. Krogh, "A Matrix Model for Heterotic Spin(32)/Z2 and Type I String Theory", Nucl. Phys. B541, 87-97, (1999), hep-th/9801034

[15] T. Banks , W. Fischer, I. R. Klebanov and L. Susskind, "Schwarzschild Black Holes in Matrix Theory II ", JHEP 9801, 008, (1998), hep-th/9711005

[16] T.Banks, "Matrix Theory", Nucl. Phys. Proc. Suppl. 63, 180-224, (1998), hep-th/9710231

[17] J. Polchinksi and P. Pouliot, "Membrane Scattering with M- Momentum Transfer" Phys. Rev. D56, 6601-6606, (1997), hep-th/9704029

[18] N. Ohta and J. Zhou , "Euclidean Path Integral, D0- Branes and Schwarzschild Black Holes in Matrix Theory", Nucl. Phys. B522, 125-136, (1998), hep-th/9801023

19] O. Tafjord and V. Periwal, "Finite- Time Amplitudes in Matrix Theory", Nucl. Phys. B517, 227-238, (1998), hep-th 9711046

[20] A. A. Tseytlin and I. Chepelev, "Interactions of type IIB D- Branes from D- instanton matrix model", Nucl. Phys. B511, 629-646, (1998), hep-th/9705120

21] W. Taylor and D. Kabat, "Spherical membranes in Matrix theory", Adv. Theor. Math. Phys. 2, 181-206, (1998), hepth/9711078

[22] W. Taylor and D. Kabat, "Linearized supergravity from Matrix theory", Phys. Lett. B426, 297-305, (1998), hep-th/9712185

[23] Taylor, "D- brane field theory on compact spaces" Phys. Lett B394, 283-287, (1997), hep-th/9610042

[24] E. Martinec and M. Li, "Probing Matrix Black Holes", hep-th/9801070 